Cardiology and Angiology: An International Journal
3(1): 1-11, 2015, Article no.CA.2015.001
ISSN: 2347-520X
SCIENCEDOMAIN international
wWw.SCiencedomain.org

\title{
Atypical Cardiac Autonomic Neuropathy Identified with Entropy Measures
}

\author{
David J. Cornforth ${ }^{1,2^{\star}}$ and Herbert F. Jelinek ${ }^{3,4}$ \\ ${ }^{1}$ Applied Informatics Research Group, University of Newcastle, Callaghan NSW 2308, Australia. \\ ${ }^{2}$ School of Engineering and Information Technology, University of New South Wales, Australian \\ Defence Force Academy, Canberra, Australia. \\ ${ }^{3}$ Centre for Research in Complex Systems, School of Community Health, Charles Sturt University, \\ Albury, Australia. \\ ${ }^{4}$ Australian School of Advanced Medicine, Macquarie University, Sydney, Australia.
}

\begin{abstract}
Authors' contributions
This work was carried out in collaboration between all authors. Author DJC designed the study, performed the statistical analysis, and drew the graphs. Author HFJ recruited the participants, collected data and managed the literature searches. All authors contributed to the writing of the manuscript and read and approved the final manuscript.

Article Information

DOI: $10.9734 / C A / 2015 / 12524$ Editor(s):

(1) Anonymous Reviewers:

(1) Abdulrahman M. Almoghairi, Adult Cardiology Department, Prince Sultan Cardiac Center, Riyadh, Saudi Arabia. (2) lana Simova, Department of Noninvasive Cardiovascular Imaging and Functional Diagnostics, National Cardiology Hospital, Bulgaria. Peer review History: http://www.sciencedomain.org/review-history.php?iid=641\&id=26\&aid=5971
\end{abstract}

\section{ABSTRACT}

Aims: To identify Cardiac Autonomic Neuropathy (CAN) from a range of measures extracted from Heart Rate Variability (HRV), including higher moments of RR intervals and a spectrum of entropy measures of RR intervals.

Study Design: Analysis of HRV measured from participants at a diabetes screening clinic. Groups were compared using t-tests to identify variables that provide separation between groups.

Place and Duration of Study: Charles Sturt Diabetes Complications Clinic, Albury, NSW Australia. Methodology: Eleven participants with definite CAN, 67 participants with early CAN, and 71 without CAN had their beat-to-beat fluctuations analyzed using two spectra of HRV: the spectrum of moments of RR intervals and the spectrum of Renyi entropy measures. RR intervals were extracted from ECG recordings and were detrended before analysis. 
Results: Higher moments of RR intervals identified a previously unnoticed sub-group of patients who are atypical within the definite CAN group. Classification of CAN progression was better with Renyi entropy measures than with moments of RR intervals. Significant differences between early and definite CAN were found with the sixth and eighth moments, $(P=.022$ and $P=.042$ respectively), but for entropy measures $P$ values were orders of magnitude smaller.

Conclusion: Identification of early CAN provides the opportunity for early intervention and better treatment outcomes, as well as identifying atypical cases. Our findings illustrate the value of exploring a range of different measures when attempting to detect differences in groups of patients with CAN.

Keywords: Cardiac autonomic neuropathy; cardiac arrhythmia; heart rate variability; entropy measures.

\section{INTRODUCTION}

Cardiovascular disease (CVD) and sudden cardiac death (SCD) represent a major portion of world-wide morbidity and mortality. In the United States, the incidence of SCD has been reported at 300,000 annually, but may be higher as the exact definition of SCD remains to be clarified [1]. In addition, an aging population and higher rates of obesity and diabetes may lead to an increase in SCD, which is associated with autonomic nervous system dysregulation of the heart $[2,3]$. Coronary artery disease is a multifactorial disease that is a major contributor to SCD, as are congestive heart failure, left ventricular dysfunction and post-myocardial infarction $[4,5]$. Accurate, non-invasive, clinical diagnostic tools have the potential to reduce the incidence of SCD in at-risk populations. Autonomic nervous system modulation of the heart leads to variability in the heart rate and in the length of the inter-beat interval. A certain degree of beat-to-beat fluctuation is an important physiological attribute, and a loss of variability in heart rate is associated with pathophysiology and increased risk of adverse cardiac events. Thus an increased heart rate or lowered heart rate variability (HRV) have been validated as markers of increased risk of myocardial infarct $[4,6]$.

Cardiovascular function is under the modulation of the autonomic nervous system. Damage to the parasympathetic or sympathetic part of the autonomic nervous system leads to dysfunction of heart rate control and vascular dynamics, and an increased risk of mortality, as shown in the ACCORD trial [7]. Cardiac Autonomic Neuropathy (CAN) has been described in diabetes, Parkinson's disease, depression, coronary heart disease and congestive heart failure [8-12]. CAN is a disease that involves nerve damage leading to increasingly abnormal control of heart rate, which is especially prominent in people with diabetes. The extent of the loss of sympathetic and parasympathetic involvement in regulating the heart rate can be determined from an ECG recording and analyzed to provide a risk stratification tool.

The standard clinical test for CAN is the Ewing battery, but this has limitations, as one or more of the five tests may be contraindicated due to cardiac or respiratory disease [13-15]. Analysis of the distribution of RR intervals over a selected period such as 20 minutes provides a more robust basis for determining autonomic nervous system function [16]. The simplest characterisation of heart rate variability remains the mean heart rate and standard deviation; however other measures may provide further insight.

\subsection{Heart Rate Variability}

A common type of ECG signal is shown in (Fig. 1). Such signals have been studied extensively and the diagnostic value of the different features is well established. Letters are used to identify ECG features. The fiducial point or peak of the QRS interval can be identified most easily and is therefore used to obtain an RR interval tachogram, from which the heart rate variability can be obtained. The natural rhythm of the human heart is known to vary in response to sympathetic and parasympathetic signals. Generally, sympathetic activity increases HR and decreases HRV, whereas parasympathetic activity decreases HR and increases HRV [17]. HRV is commonly used in assessing the regulation of cardiac autonomic function $[18,10]$. 


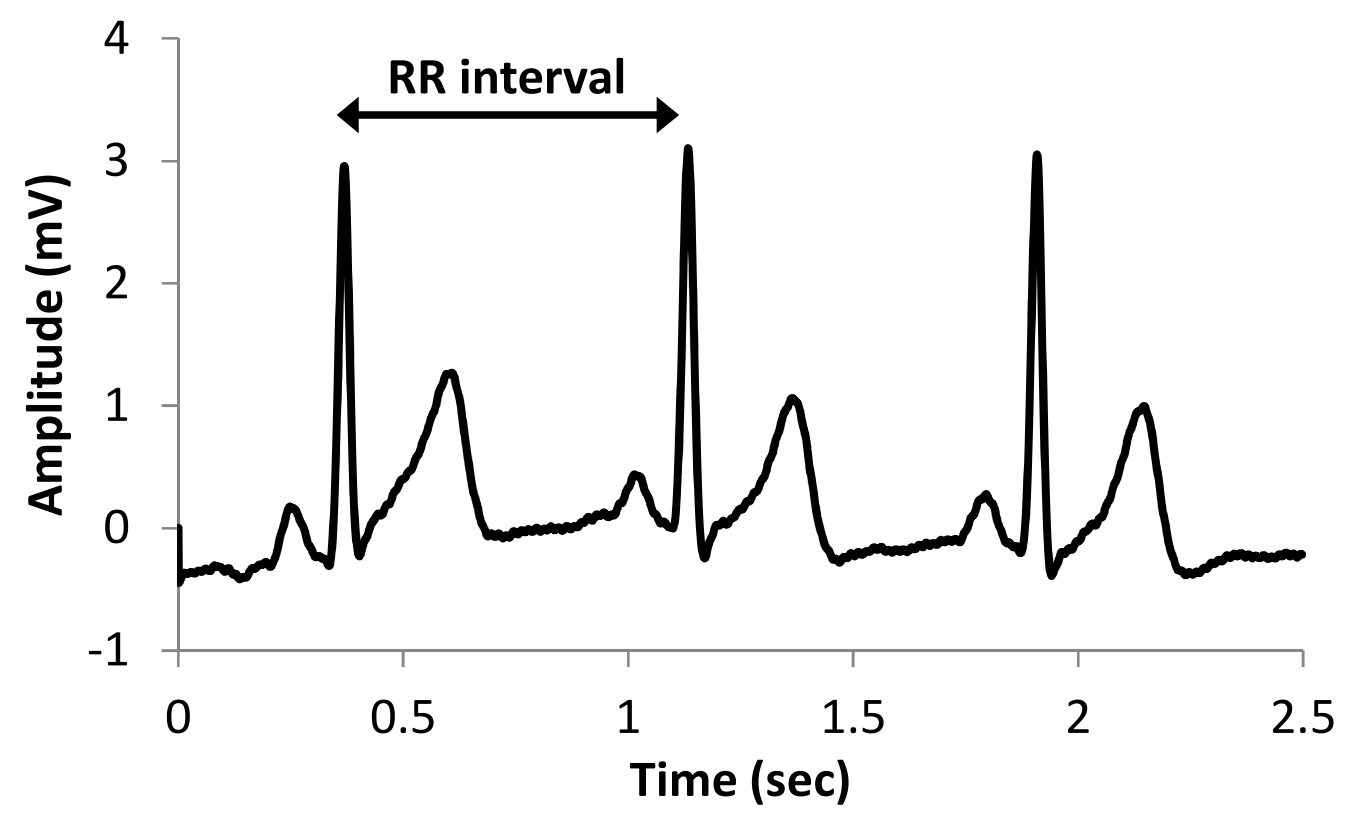

Fig. 1. A typical ECG signal showing the RR Interval

The ECG signal may often be degraded by the presence of noise, so that the most reliable feature that can be obtained from low quality recordings (and therefore the most easily obtained measurement) is the interval between successive $R$ peaks, the RR interval, which is the inverse of the heart rate. A typical adult heart rate is $60-80$ beats per minute, with typical RR interval lengths between 750 and 1000 milliseconds. RR intervals can be subjected to further analysis through a variety of algorithms in order to provide measures with good discriminant power, based on the difference of RR interval variability. HRV provides information only on the changes in the interval length, is non-invasive and easy to obtain from an ECG recording.

Cardiac Autonomic Neuropathy (CAN) leads to arrhythmias and may precipitate SCD. An open question is to what extent this condition is detectable by measures based on HRV. An even more desirable option is to detect CAN in its early, preclinical stage, to improve treatment and treatment outcomes.

\subsection{Multi-scale Moments}

Moments are measures of distribution such as mean, median, mode, skewness and kurtosis. The various moments from $R R$ intervals provide a numeric value by which the distribution can be characterized. The familiar arithmetic mean and variance of $R R$ intervals can be informally viewed as moments of order 1 and 2 respectively, where order refers to the exponent used in calculating these values. Higher order moments can be defined as:

$$
m_{k}=E\left[(X-\mu)^{k}\right]
$$

where $E[X]$ is the expectation of $X$, and $\mu$ is the arithmetic mean of the variable $X$. Expectation is commonly interpreted as the sum of observations on $X$ in a sample of size $n$, divided by $n$, so that for example the second moment or variance is defined as:

$$
s^{2}=\frac{1}{n} \sum_{i=1}^{n}\left(x_{i}-\bar{x}\right)^{2}
$$

which calculates deviation in observations $x_{i}$ in the sample of size $n$ from the mean. The third and fourth moments have a known interpretation, as the Skewness and Kurtosis respectively, although $m_{3}$ and $m_{4}$ are usually subject to corrections in order to address statistical bias and magnitude. Skewness describes the amount of asymmetry of the distribution, so can reveal whether the distribution is leaning to the left or right, and consequently whether the tails are larger on the lower or upper sides of the distribution. A negative value indicates a larger 
tail for values lower than the mean, while a positive value indicates a larger tail above the mean. Kurtosis measures the flatness of the distribution. A flat or platykurtic distribution has a negative value for kurtosis, while a peaked or leptokurtic distribution has a sharp peak. The former indicates that the variance of the distribution is due to unusually large deviations from the mean, when compared to a Gaussian distribution. The latter indicates that the variance is due to frequent small deviations.

Higher moments have more difficult interpretations, but provide different measures of the distribution of RR intervals, so can be used to compare different groups of patients. It is usual to normalize these moments to provide a scale invariant spectrum:

$$
\mu_{k}=\frac{m_{k}}{\sigma^{k}}
$$

where $\mu_{k}$ is the standardized moment, and $\sigma^{k}$ is the standard deviation raised to the power of $k$.

\subsection{Multiscale Renyi Entropy}

In the context of the analysis of heart rate variability, various entropy measures can estimate the variability of the HRV. An entropy measure is typically of the form:

$$
H(X)=-\sum_{i=1}^{n} p\left(x_{i}\right) \log _{b} p\left(x_{i}\right)
$$

where $p\left(x_{i}\right)$ is the probability of the random variable $x$, and $b$ is the base of the logarithm, commonly 2 . Renyi entropy $H$ is a generalization of the Shannon entropy:

$$
H_{\alpha}(X)=\frac{1}{1-\alpha} \log _{2}\left(\sum_{i=1}^{n} p_{i}^{\alpha}\right)
$$

where $p_{i}$ is the probability that $X=x$ and $\alpha$ is the order of the entropy measure. This is the parameter that is varied to produce the multiscale entropy. The probability can be estimated in a number of ways. In this work we estimate the probability of a sequence of $\mathrm{RR}$ intervals of length $\pi$ by comparing the sample $i$ with all other samples of length $\pi$ in the recording, using methods similar to those used to estimate sample entropy, and as outlined by Lake [19]. This involves measuring the distance between sample $i$ and all other samples $j$, then estimating $p_{i}$ using a Gaussian (normal) kernel:

$$
p_{i}=\sum_{j=0}^{n} \exp \left(\frac{-d i s t_{i j}^{2}}{2 \sigma^{2}}\right)
$$

where $\sigma$ is a parameter controlling the width of the density function and dist $t_{i j}$ is a distance measure, in this case Euclidean, in $\pi$ dimensions:

$$
\operatorname{dist}_{i j}=\sum_{k=0}^{\pi}\left(x_{i+k}-x_{j+k}\right)^{2}
$$

This yields a probability estimate for each sample of length $\pi$, with the desirable property that its value lies between 0 and 1 .

\section{METHODOLOGY}

Anthropometric and clinical data were obtained from patients reviewed at the Charles Sturt Diabetes Complications Screening Group (DiScRi), Australia [20]. Participants attending the screening clinic had their lead II ECG recorded for 20 minutes and $R R$ intervals analysed. The subjects were comparable for age, gender, and heart rate, and the same physical conditions were used for each subject. ECGs were recorded using a Maclab Pro with Chart 7 software (AD Instruments, Sydney). Initial screening of participants led to the exclusion of those with heart disease, presence of a pacemaker, kidney disease or polypharmacy including multiple anti-arrhythmic medications. The study was approved by the Charles Sturt University Human Ethics Committee and written informed consent was obtained from all participants. CAN was defined using the Ewing battery criteria, and so participants were separated into early CAN, definite CAN, or no CAN $[13,21,22]$.

Eleven participants with definite CAN, 67 participants with early CAN, and 71 without CAN attending the screening clinic participated. From the 20-minute recording, a 15-minute segment was taken from the middle of the original recording to remove start-up artefacts and movement artefacts at the end of the recording. Only the RR intervals were retained, and no other information from the ECG were utilised in this study. The baseline was removed by 
subtracting the mean value of the RR interval from the RR data. The trend was removed after analysis by linear correlation. For each detrended series the mean, variance, and higher moments were calculated as described above.

The multi-scale Renyi Entropy was calculated from $-5<\alpha<+5$, where $\alpha$ represents the scaling exponent and $\alpha=1$ is the Shannon entropy. For all calculated measures, a student's t-test was performed to compare the means of every variable. For all variables from the moments and the Renyi spectra, histograms were calculated and smoothed using a filter of:

$$
f_{i}^{*}=\frac{1}{9}\left(f_{i-2}+2 f_{i-1}+3 f_{i}+2 f_{i+1}+f_{i+2}\right)
$$

Frequency values for all histograms were then normalised, by dividing by the number of patients in each class. A selection of these histograms is presented below.

\section{RESULTS AND DISCUSSION}

Results for the spectrum of moments are shown in (Table 1). Column headings are given for each calculated moment. The first two columns provide the mean and variance. Under these headings, the $P$-value results of t-tests are provided, comparing the means of the three patient groups for each moment. Any value below $P=.05$ is regarded as significant at the $95 \%$ confidence interval level, and is indicated by shading.

(Table 1) shows there is evidence for a difference between the mean of RR intervals $(P \leq 4.99 E-6)$, and evidence of a difference between variance of $R R$ intervals $(P \leq 8.53 E-4)$. These results are well known and agree with the findings of previous studies [23]. Also of clinical interest is the significant difference between Early and Normal CAN groups for the sixth and eighth moments $(P=.022$ and $P=.042$ respectively).

Table 1. $P$ values comparing mean value of moments for each of the 3 classes: $D:$ Definite CAN, E: Early CAN and N: Normal (controls). Skewness is closely related to $\mu 3$, while kurtosis is closely related to $\mu 4$. Shaded cells indicate significance at $P=.05$ or better

\begin{tabular}{llllllllll}
\hline T-test & Mean & Vari & $\boldsymbol{\mu 3}$ & $\boldsymbol{\mu 4}$ & $\boldsymbol{\mu 5}$ & $\boldsymbol{\mu 6}$ & $\boldsymbol{\mu 7}$ & $\boldsymbol{\mu 8}$ & $\boldsymbol{\mu 9}$ \\
\hline D vs. E & $5.0 \mathrm{E}-6$ & $8.5 \mathrm{E}-4$ & .63 & .89 & .17 & .83 & .16 & .53 & .15 \\
E vs. N & $5.6 \mathrm{E}-7$ & $9.1 \mathrm{E}-6$ & .74 & .063 & .29 & .022 & .23 & .042 & .28 \\
N vs. D & $4.7 \mathrm{E}-10$ & $1.8 \mathrm{E}-7$ & .84 & .32 & .41 & .24 & .44 & .22 & .055 \\
\hline
\end{tabular}

The values of mean and variance for the three patient groups are illustrated using smoothed histograms in (Figs. 2 and 3). Patients in the Normal group (controls) have lower mean RR interval (Fig. 2) and higher variance (Fig. 3), while patients in the Definite group (confirmed CAN) have a higher mean and lower variance. The values of the $6^{\text {th }}$ and $8^{\text {th }}$ moments are illustrated similarly in (Figs. 4 and 5). Examination of these two figures reveals a hitherto unnoticed outlier sub-group in the Definite CAN group, which is apparent in both (Figs. 4 and 5). This outlier sub-group consists of two patients who have elevated values, apparent in higher even moments including the $4^{\text {th }}$ moment (not shown) but not apparent from any of the odd moments analysed. This difference is due to the exponents treat both positive and negative deviations from the mean as equivalent. Moments calculated using odd exponents on the other hand, treat deviation from the means differently, depending on whether they are positive or negative. These outliers were not detected by the mean or variance, but became apparent when higher moments were examined. This highlights the value of exploring higher moments associated with the RR interval distribution for analysis of HRV.

The results for Renyi entropy are shown in (Table 2). Column headings identify the Renyi entropy calculated for different values of the exponent $\alpha$. As in the previous table, the $P$ values resulting from t-tests are provided below these headings. An examination of significant results, in shaded cells, reveals very different results for negative and positive values of $\alpha$. Nearly all the significant values correspond to $\alpha<0$. There is little difference resulting from the actual value of $\alpha$ chosen, but in general the negative part of the Renyi spectrum appears to provide superior discrimination between patient groups. fact that moments calculated using even 
Table 2. $P$ values comparing mean of renyi entropy for each of the 3 classes: D: Definite CAN, E: Early CAN and N: Normal (controls). Shaded cells indicate significance at $P=.05$ or better

\begin{tabular}{lllllllllll}
\hline T-test & $\mathbf{H}(-5)$ & $\mathbf{H}(-4)$ & $\mathbf{H}(-3)$ & $\mathbf{H}(-2)$ & $\mathbf{H}(-\mathbf{1})$ & $\mathbf{H}(\mathbf{1})$ & $\mathbf{H}(\mathbf{2})$ & $\mathbf{H}(\mathbf{3})$ & $\mathbf{H}(\mathbf{4})$ & $\mathbf{H}(\mathbf{5})$ \\
\hline D vs. E & .15 & .16 & .17 & .18 & .22 & .27 & .25 & .22 & .21 & .20 \\
E vs. N & $7.7 \mathrm{E}-5$ & $7.0 \mathrm{E}-5$ & $6.1 \mathrm{E}-5$ & $5.3 \mathrm{E}-5$ & $7.2 \mathrm{E}-5$ & .09 & .19 & .22 & .21 & .20 \\
N vs. D & $8.9 \mathrm{E}-5$ & $1.0 \mathrm{E}-4$ & $1.3 \mathrm{E}-4$ & $2.2 \mathrm{E}-4$ & $7.4 \mathrm{E}-4$ & .057 & .066 & .060 & .054 & .050 \\
\hline
\end{tabular}

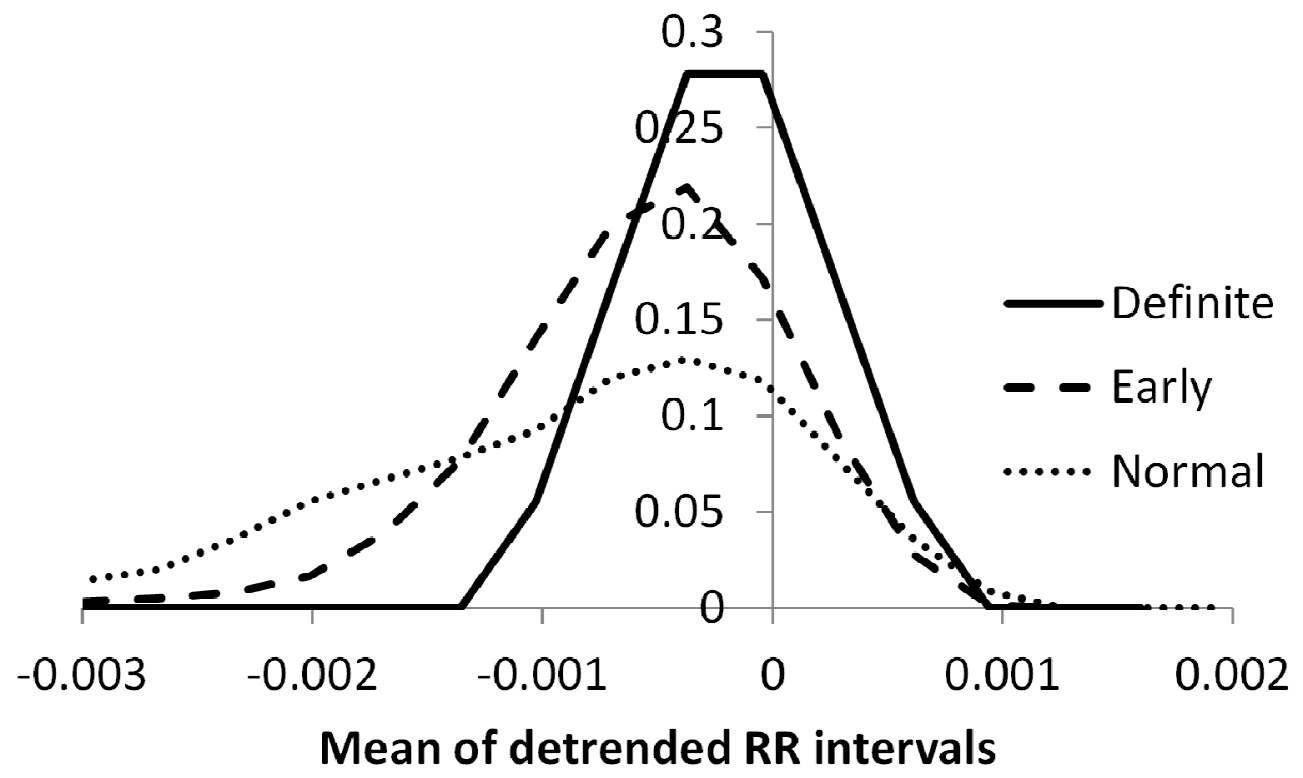

Fig. 2. Smoothed histogram comparing the three patient groups for mean RR interval

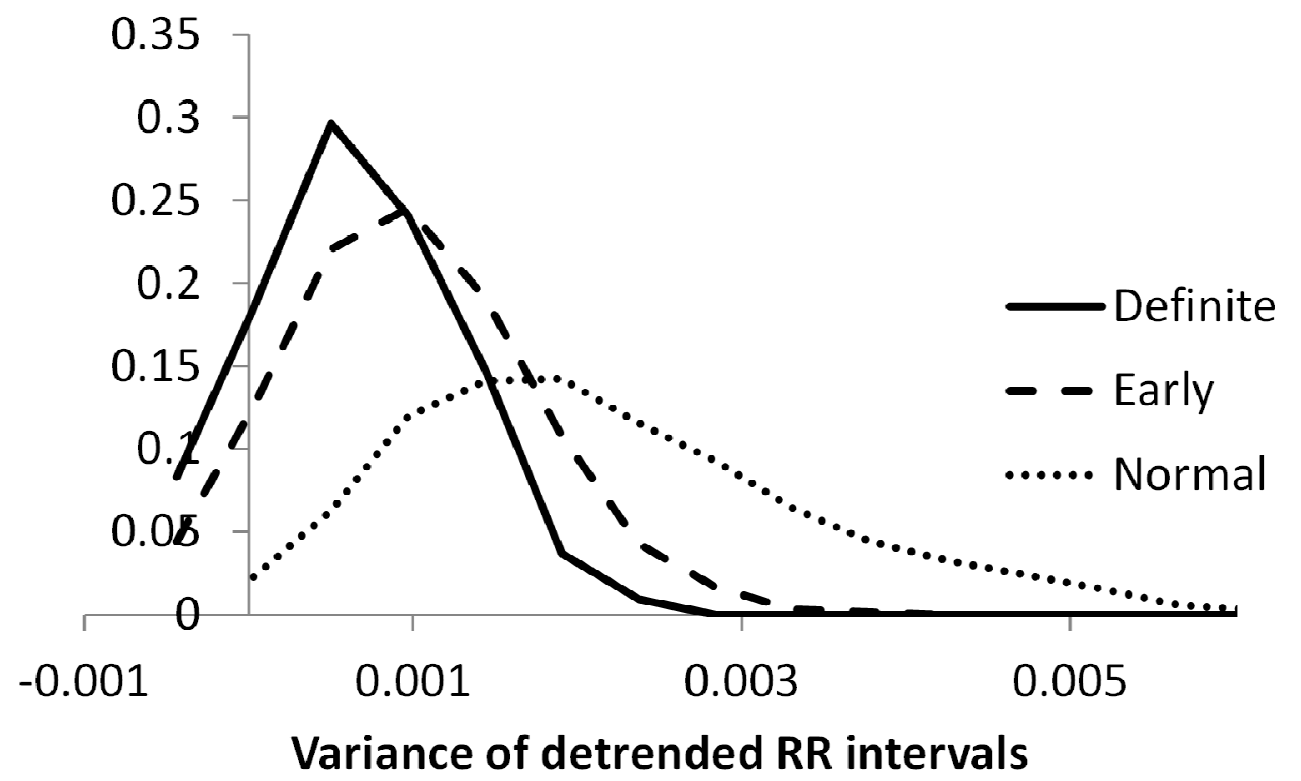

Fig. 3. Smoothed histogram comparing the three patient groups for variance of RR interval 


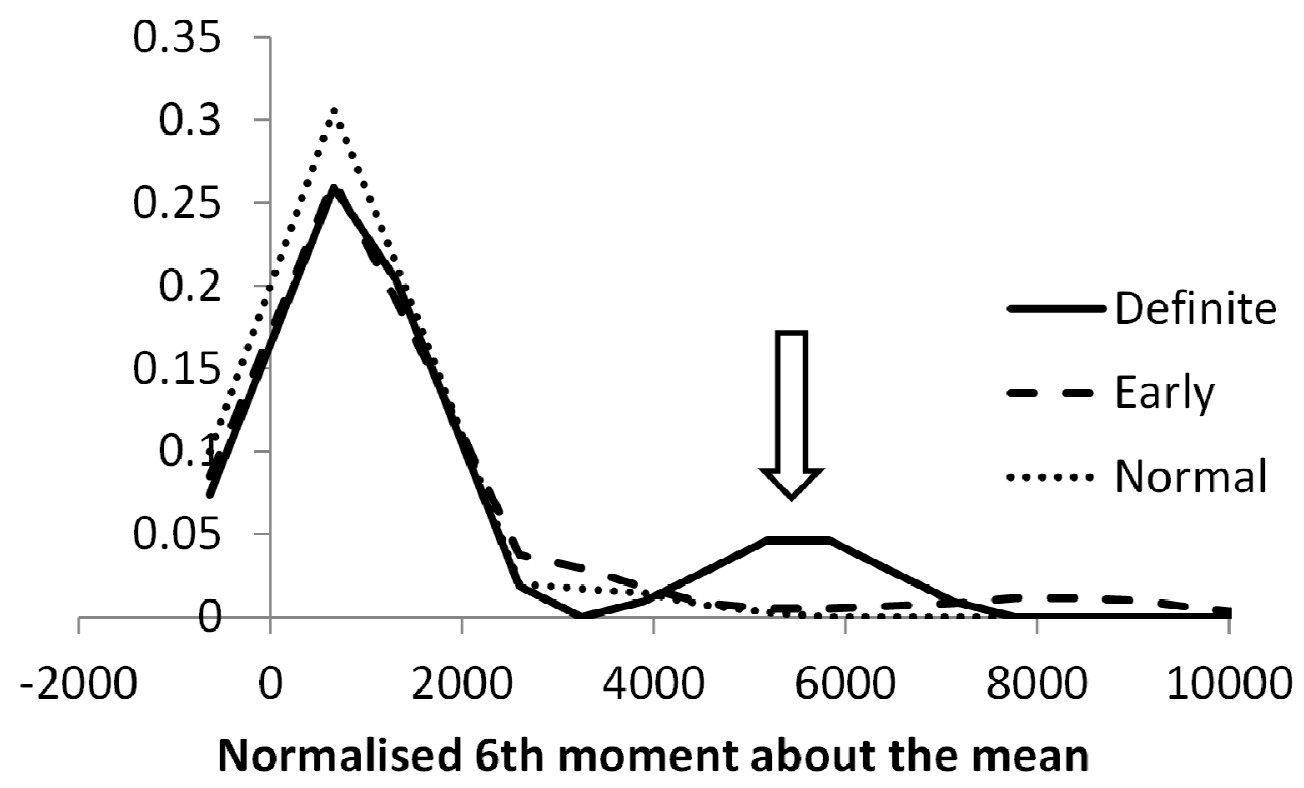

Fig. 4. Smoothed histogram comparing the three patient groups for the 6th moment. This reveals a previously undetected outlier sub-group (indicated with arrow) in the definite group

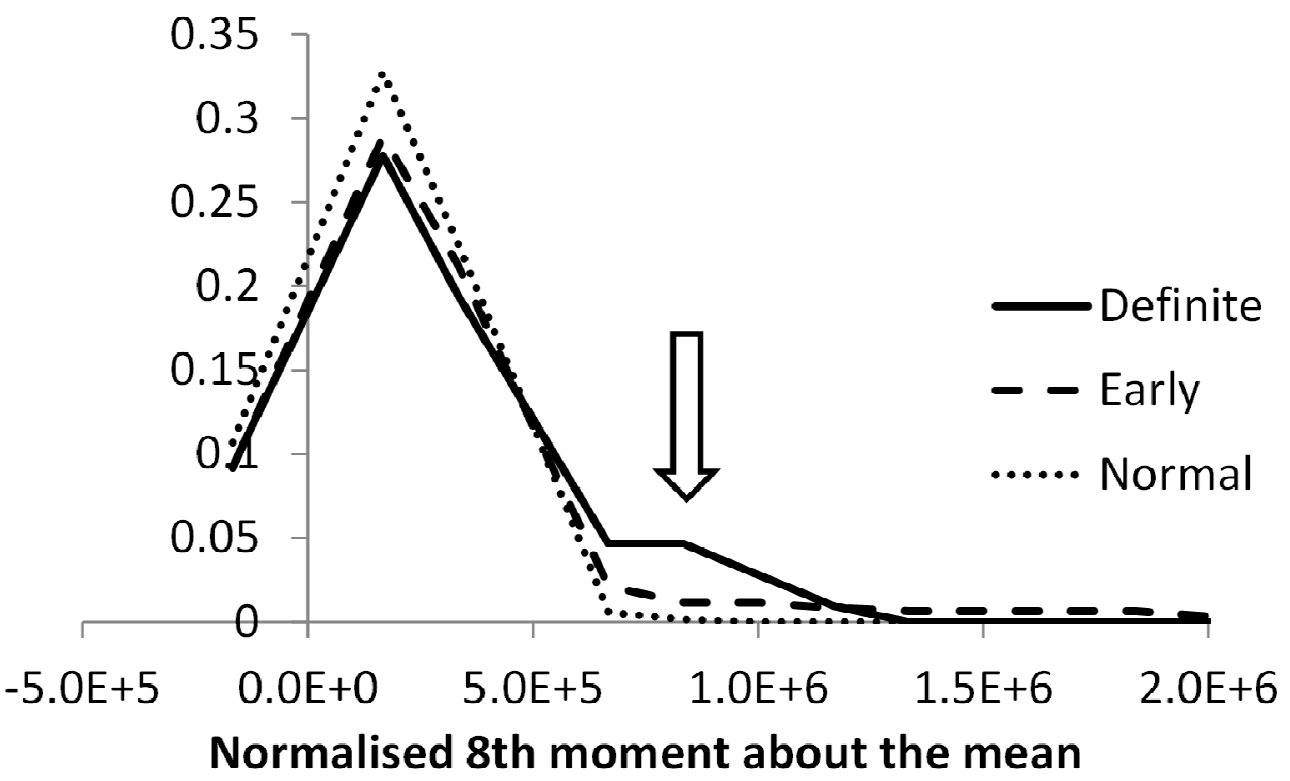

Fig. 5. Smoothed histograms comparing the three patient groups for the 8th moment. The previously undetected outlier sub-group is indicated with an arrow

The smoothed histogram for Renyi entropy with $\alpha=-5$ is shown in (Fig. 6). The differences are readily observed, with patients from the Definite group providing, on average, a higher value of Renyi entropy (mean of 2.14), followed by patients from the Early group (mean of 2.06).
The lowest values for $H(-5)$ were obtained from patients in the Normal CAN group, with a mean value of 1.88. Compare this with the smoothed histogram for the Shannon entropy $H(1)$ shown in (Fig. 7). Here the three patient groups cannot be distinguished from each other. It is clear that the 
Shannon entropy is unable to distinguish between the three groups of patients, whereas the Renyi entropy with negative exponents is able to separate these groups.

In this study, we have examined two spectra of measures. The spectrum of moments is obtained by extending the variance using exponents greater than 2. These moments include skewness and kurtosis, but form part of a spectrum, which extends further to include moments of order 8 and higher. For instance the third moment (or skewness) indicates whether the variance is due to fewer, larger deviations on one side of the distribution compared to the other. In definite CAN when the sympathetic component of autonomic regulation starts to predominate or parasympathetic withdrawal is occurring, a skewed distribution favouring shorter $\mathrm{RR}$ intervals can be expected. The current work indicates that the distributions are fairly symmetrical, as shown by relatively low values for the third moment (skewness) and values that were similar across all three patient groups. Kurtosis describes the flatness of the distribution relative to the normal distribution. $R R$ interval time series with high kurtosis have a distinct peak near the mean, decline rather rapidly, and have heavy tails. In this case the relatively large values for the $4^{\text {th }}$ moment indicate a distribution with a high peak around the mean, indicating that most of the variance is due to many relatively small deviations of the RR interval size from the mean, and very few large deviations.

The spectrum of moments higher than of order 4 for $R R$ intervals suggested that higher odd numbered moments do not afford a measure to assist in distinguishing the three groups. However, the higher even moments drew attention to a sub-group of patients who are atypical within the group with definite CAN. Moments with even exponent treat positive and negative deviations from the mean in a similar way, so may group together values that may not be associated using odd moments. This subgroup requires further investigation. However it is not uncommon to misclassify a patient using the Ewing battery, especially if only one or two of the required five tests are used. For the current study, only those ECGs were analysed where results for the complete Ewing battery of tests was available. In spite of this, some misclassification is possible. In addition, patients with cardio respiratory disorders, those that are frail or obese may have difficulty in performing the required tests. Therefore passive testing for $\mathrm{CAN}$, as is the case by interpreting the RR intervals obtained from an ECG recorded at rest, may provide more robust results for assessment of CAN progression.

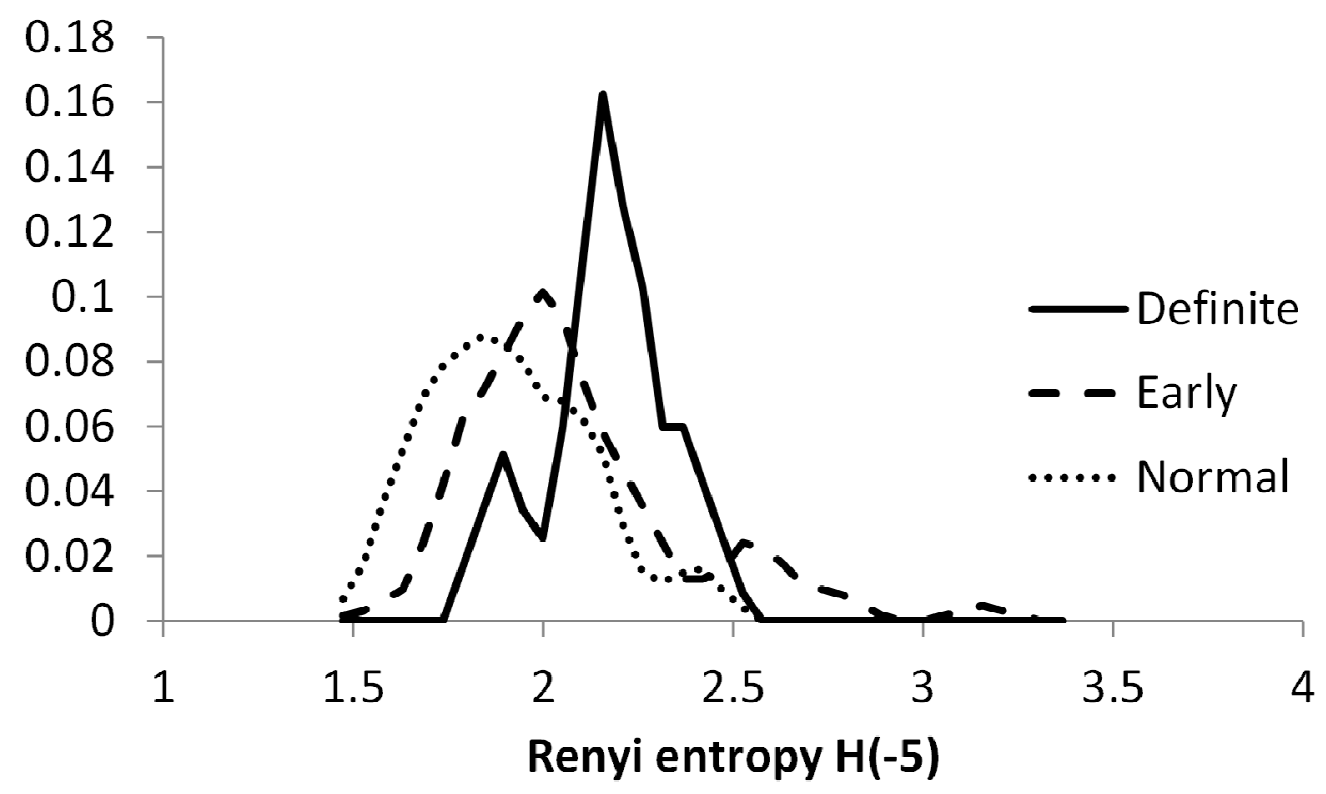

Fig. 6. Smoothed histograms comparing the three patient groups for renyi entropy with $\alpha=-5$ 


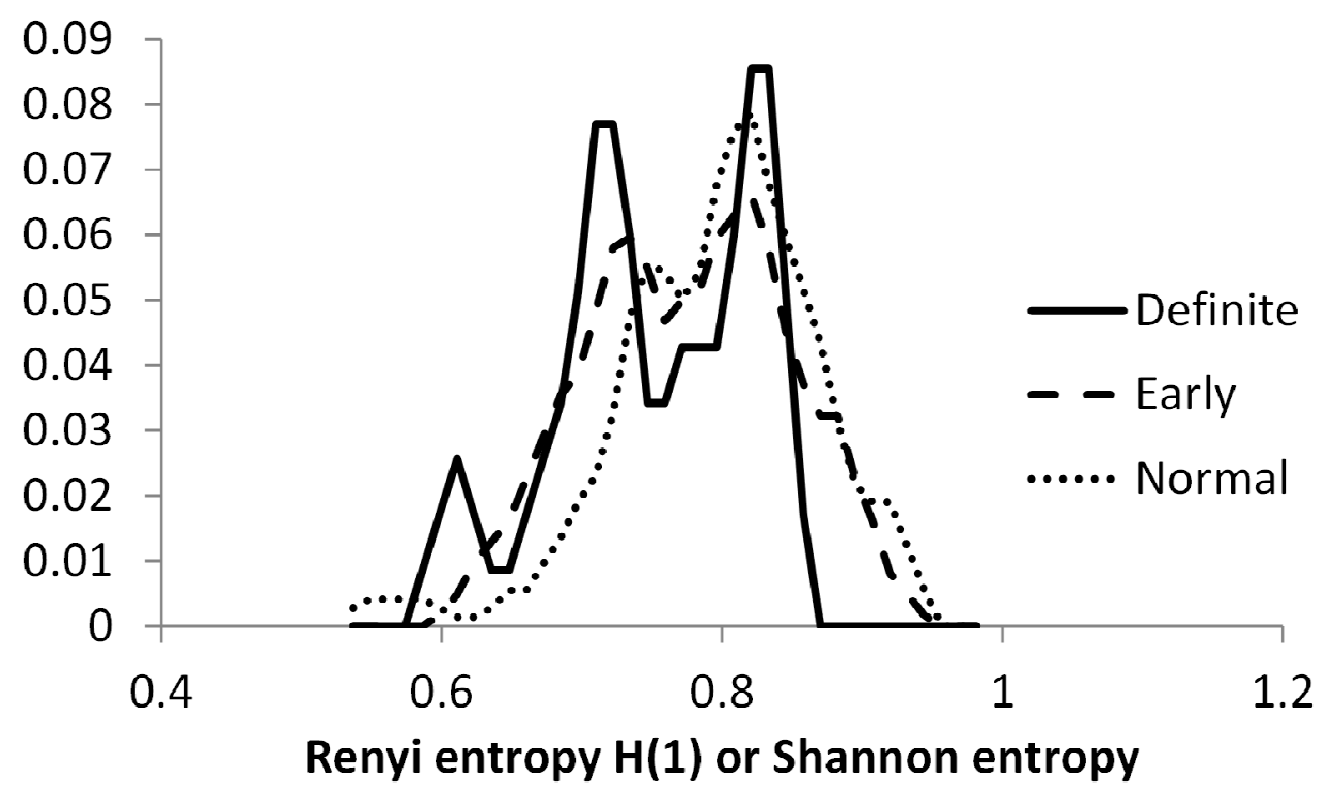
Fig. 7. Smoothed histograms comparing the three patient groups for Renyi entropy with $\alpha=1$
(equivalent to Shannon entropy)

The spectrum of Renyi entropy was much more successful in distinguishing patient groups, showing highly significant differences in means for the three groups $(P<.0001)$. However, the use of a spectrum of measures revealed that these differences could not be detected using the Shannon entropy $(\alpha=1)$. It was necessary to explore more fully the range of possible exponents in order to discover a suitable entropy measure that could distinguish patient groups. One drawback of the study was that groups such as those with diabetes or obesity often have prescribed medication, which may directly or indirectly affect cardiac function and therefore rhythm analysis. Our data reflect this, as the number of participants identified with definite CAN is rather small due to the exclusion criteria applied.

\section{CONCLUSION}

Risk stratification of sudden cardiac death is an important component in clinical practice, especially in patients with diabetes, where the risk is much higher and an asymptomatic stage associated with Cardiac Autonomic Neuropathy (CAN) often occurs. It is desirable to find a relatively non-invasive method to identify CAN, and this work explores the feasibility of identification based on measures of Heart Rate Variability (HRV). In this work, we have examined the use of two spectra of measurements to identify CAN: the spectra of moments and the spectra of Renyi entropy, both calculated from $R R$ intervals.

The mean and variance of the $R R$ interval are useful discriminators, but higher moments did not provide any additional discriminating power, except that some moments were able to detect outliers. However the Renyi spectrum, in particular the negative part, was consistently successful in identifying groups of patients.

Our findings illustrate the value of exploring a range of measures when attempting to detect differences in groups of patients. Although measures such as mean, variance and Shannon entropy may be more well-known than Renyi entropy, these measures may not provide the required discrimination. An exploration of multiscale measures as demonstrated in this study provides new insights into cardiovascular disease.

\section{CONSENT}

All authors declare that written informed consent was obtained from the patients for publication of the study.

\section{ETHICAL APPROVAL}

All authors hereby declare that all experiments have been examined and approved by the Charles Sturt University Human Ethics 
Committee and have therefore been performed in accordance with the ethical standards laid down in the 1964 Declaration of Helsinki.

\section{ACKNOWLEDGEMENTS}

The authors would like to thank Bev de Jong for technical assistance and Roche Australia Pty for providing consumables for blood glucose measurements. The study was funded in part by a Compacts Funding from Charles Sturt University. Roche Australia Pty and Charles Sturt University played no role in the design, data collection, analysis and interpretation of the study.

\section{COMPETING INTERESTS}

Authors have declared that no competing interests exist.

\section{REFERENCES}

1. Myerburg RJ. Sudden cardiac death: Exploring the limits of our knowledge. J Cardiovasc Electrophysiol. 2001;12(3):369-381.

2. Friedman GD, Klatsky AL, et al. Predictors of sudden cardiac death. Circulation. 1975;52(6):164-169.

3. Sexton PT, Walsh J, et al. Risk factors for sudden unexpected cardiac death in Tasmanian men. Aust NZ J Med. 1997;27:45-50.

4. Priori SG, Aliot E, et al. Task force on sudden cardiac death of the European society of cardiology. Eur Heart J. 2001;22(16):1374-1450.

5. Zheng ZJ, Croft JB, et al. Sudden cardiac death in the United States, 1989 to 1998. Circulation. 2001;104(18):2158-2163.

6. La Rovere MT. Heart rate and arrhythmic risk: Old markers never die. Europace. 2010;12(2):155-157.

7. Pop-Busui R, Evans GW, et al. The ACCORD study group. Effects of cardiac autonomic dysfunction on mortality risk in the action to control cardiovascular risk in diabetes (ACCORD) trial. Diabetes Care. 2010;33(7):1578-1584.

8. Kemp AH, Quintana DS, et al. Heart rate variability in unmedicated depressed patients without comorbid cardiovascular disease. Plos ONE. 2012;7(2):e30777.

9. Voss A, Schroeder R, et al. Segmented symbolic dynamics for risk stratification in patients with ischemic heart failure. Cardiovascular Engineering and Technology. 2010;1(4):290-298.

10. Vinik Al, Erbas $T$, et al. Diabetic cardiac autonomic neuropathy, inflammation and cardiovascular disease. Journal of Diabetes Investigation. 2013;4(1):4-8.

11. Salvi $V$, Hingorani $P$, et al. Prediction of mortality using measures of cardiac autonomic dysfunction in the diabetic and non diabetic population: The MONICA/KORA Augsburg Cohort Study. Diabetes Care. 2008;31(10):e74.

12. Siddiqui MF, Rast $S$, et al. Autonomic dysfunction in Parkinson's disease: A comprehensive symptom survey. Parkinsonism \& Related Disorders. 2002;8(4):277-284.

13. Ewing DJ, Martyn CN, et al. The value of cardiovascular autonomic functions tests: 10 years' experience in diabetes. Diabetes Care. 1985;8(5):491-498.

14. Jelinek HF, Kelarev AV, et al. Rule-based classifiers and meta-classifiers for identification of cardiac autonomic neuropathy progression. International Journal of Information Science and Computer Mathematics. 2012;5(2):49-53.

15. Stranieri A, Abawajy J, et al. A decision tree approach for ewing test selection to support the clinical assessment of cardiac autonomic neuropathy. Australian Datamining Conference Aus DM; 2012.

16. TFESC/NASPE. Heart rate variability. Standards of measurement, physiological interpretation, and clinical use. Task Force of the European Society of Cardiology and the North American Society of Pacing and Electrophysiology. European Heart Journal. 1996;17(3):354-381.

17. Berntson GG, Bigger JT Jr, Eckberg DL, et al. Heart rate variability: Origins, methods, and interpretive caveats. Psychophysiol. 1997;34(6):623-48.

18. Flynn AC, Jelinek HF, Smith MC. Heart rate variability analysis: $A$ useful assessment tool for diabetes associated cardiac dysfunction in rural and remote areas. Aust J Rural Health. 2005;13:77-82.

19. Lake DE. Renyi entropy measures of heart rate Gaussianity, IEEE Transactions on Biomedical Engineering. 2006;53(1).

20. Jelinek HF, Wilding C, Tinley P. An innovative multi-disciplinary diabetes complications screening programme in a rural community: A description and preliminary results of the screening. 
Australian Journal of Primary Health. 2006;12:14-20.

21. Javorka M, Trunkvalterova Z, Tonhajzerova I, et al. Short-term heart rate complexity is reduced in patients with type 1 diabetes mellitus. Clin. Neurophysiol. 2008;119:1071-81.

22. Khandoker $\mathrm{AH}$, Jelinek HF, Palaniswami M. Identifying diabetic patients with cardiac autonomic neuropathy by heart rate complexity analysis. Biomed Eng Online. 2009;8:3.

23. Goldberger JJ, Challapalli S, Tung R, et al. Relationship of heart rate variability to parasympathetic effect. Circulation. 2001;103(15):1977-1983.

(c) 2015 Cornforth and Jelinek; This is an Open Access article distributed under the terms of the Creative Commons Attribution License (http://creativecommons.org/licenses/by/4.0), which permits unrestricted use, distribution, and reproduction in any medium, provided the original work is properly cited.

Peer-review history:

The peer review history for this paper can be accessed here:

http://www.sciencedomain.org/review-history.php?iid=641\&id=26\&aid=5971 